Lungu Mihaela ${ }^{1}$, Romila Aurelia ${ }^{2}$, Hangan L.T. ${ }^{3}$, Caraban B.M. ${ }^{3}$

\title{
Schizencephaly Associated With Polymicrogirya - Cause For Late-Onset Epileptic Seizures in Adult. A Case Report.
}

${ }^{1}$ Neurological Department, Emergency Clinical Hospital, Galati; Faculty of Medicine and Pharmacy, "Dunarea de Jos" University of Galati

${ }^{2}$ Geriatric Department, Emergency Clinical Hospital, Galati; Faculty of Medicine and Pharmacy, "Dunarea de Jos" University of Galati, Center for Research on Medical-Pharmaceutical

${ }^{3}$ Faculty of Medicine, University "Ovidius" of Constanta

\begin{abstract}
The article presents the case of a 61-year old female patient, with no history of neurological illness, who presents a rapid onset of two convulsive seizures, triggered by a psychological trauma. The first convulsive seizure is repeated within 24 hours.

The general, as well as the neurological clinical examination have not found any pathological signs. MRI scanning of the brain pointed to right-parietal schizencephaly, associated with polymicrogyria, the believed causes of the epileptic seizures.
\end{abstract}

Keywords: schizencephaly, polymicrogyria, late-onset epileptic seizures, MRI.

\section{Bogdan Marian Caraban}

Faculty of Medicine, University "Ovidius" of Constanta University Street, No. 1, Campus B, Constanta, Romania

email : bcaraban@yahoo.com phone: +40722322476

\section{Introduction}

The defects of cerebral sulcation producevarious degrees of impairment in neurological functions. Seizures- intractable epilepsy, delayed in development, apnea attacks are some of the neurological signs.

The failure of sulcation seems to be caused by the fact that neurons may fail to form or to migrate along glial projections, to reach the more superficial layers of the cortex, during the development of the neocortex.

So, the brain has less-convuletedgyri in the cerebral cortex, with impaired cortical laminations of neurons.

Schizencephaly is a cerebral malformation defined byYakovlev and Wadsworth as being triggered by a focal development defect in the wall of cerebral mantle, a local failure of evagination, basing his claim on finding of malformed cortex in 
the margins of the defect. Nevertheless, this may also show that the lesion precedes neural migration[1].

Levine and coworkers assignschizencephaly to a destructive, possibly ischemic lesion, occurring in the first weeks of pregnancy, when the neuronal migration is incomplete [1].

On the other side, polymicrogyria is also a malformation of cortical development, frequent enough, at an estimate prevalence of $16 \%$ in large series, associated with variable clinical and imaging features, pathological finding and various etiology [3]. It may occurs as an isolated malformation or in association with other malformation within the brain or body.It can be explained through multiple mechanisms.

One of the aforementioned mechanisms may rely on genetic errors.

Mutations in over 30 genes have been found in association with polymicrogiria, especially in the tubulin family genes.

Thus, the discovery of new genes for polymicrogiria: BiCD2, LIS-1, NDE, defect in transcription of factor ARX etc. has a major effect in pediatric neurology.

Joanna Lipka and coworkers show that neurons use cytoskeleton-based motor proteins to transport different vesicles and cellular material. The abnormalities in intracellular transport are critical factors for the degeneration and death of neurons[2].

During neuronal migration, microtubule organization and transport are controlled by PAF$\mathrm{AH}_{1} \mathrm{~B}_{1}$ ( LIS-1), DCX, YWHAE, tubulin, APOER The cytoskeleton systems, with their proteins networks govern the neuronal migration [4].

In addition, genetic defects associated with the abnormalities in intracellular transport may determined the defects of cerebral sulcation.

The genetic causes also include metabolic conditions, such as peroxisomal disorders [5].

In a few cases of this type of malformation, some congenital infection with cytomegalovirus or rubella or, also, uterus ischemia have been involved.

Despite recent genetic discoveries, the mechanism by wichpolymicrogiria cortex forms remain unknown, making the diagnostic and prenatal testing still difficult [5].

From a clinical manifestation point of view, these depend on the degree of neural damage caused by the neocortexmalforming.

Clinical manifestation appear, with varying degrees of consistency, during childhood, and consist in epileptic seizures, reduced cognitive abilities, sometimes associated with skull and facial dysmorphism: small cranium, short nose, small mandible, abnormalities in the ears or congenital heart disease. $1-$

Neuroimaging investigation- MRI is the main tool used in confirming cortical-cerebral damage.

In the following, we shall present a clinical case, diagnosed through MRI, in a 61 year old, female patient. The patient presented two generalized, lateonset, convulsive epileptic seizures.

\section{Gase Report}

C.T., a 61-year old female, with no pathologic history, checks herself into the hospital for the investigation of two late-onset epileptic seizures. The seizures manifested themselves within the last 24 hours and were of the clonic-tonic convulsive type.

The general and neurological clinical exams did not find any pathologic signs. The patient did not present any skull or facial dysmorphism, cognitive impairment or cardiac malformations.

The brain MRI revealed a rightparietal schizenchephaly, associated with rightside,parasilvianpolimicrogyria: fig. 1, 2

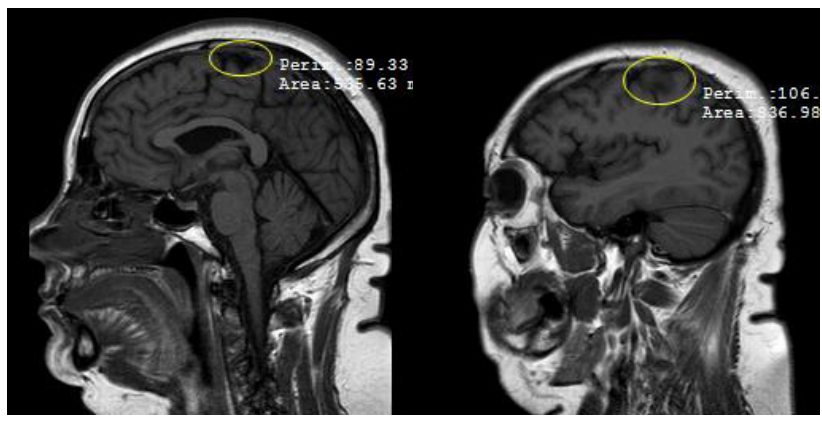

Figure 1 The Brain MRI - irregularly cerebral surface, aspect of focal cortical atrophy. 


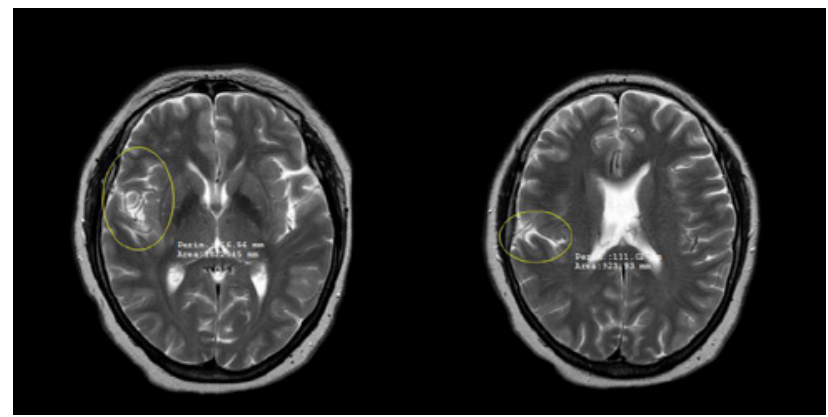

Figure 2: The brain MRI -In the right parietal side there is a schizencephaly slit, bordered by cortical matter that is close to the right lateral ventricle. On the right perisilvian side, there is a conglomerate of dysplastic gyrus (small, multiple, agglomerated). neuroimaging exam- MRI.

The malformation was considered to be a light form, not presenting any clinical manifestations until this age.

Anticonvulsive medication has been prescribed, taking into consideration the risk of recurring seizures. The patient has no symptoms and is in neurological surveillance.

\section{Acknowledgments}

Financial disclosure: none.

Informed consent: Informed consent was obtained from the patient in this case report.

\section{References}

pathological history and the fact that complementary paraclinically exams of ultrasound, blood chemistry and radiology have excluded other possible causes for the two convulsive episodes, it has been concluded that the cerebral malformation gave rise to late-onset, generalized, tonic-clonic epileptic seizures.

Taking into consideration both the brain imagery and the risk of recurring seizures, a treatment of $600 \mathrm{mg}$ per day of carbamazepin was prescribed. The patient is presently in neurological surveillance and did not repeat the convulsive seizures.

\section{Conclusions}

In this case, schizencephaly associated with polymicrogyria were considered the cause to the lateonset epileptic seizures.

The diagnosis was established through
1. Ropper, A., Samuels, M. \& Klein, J. (2014). Adams and Victor's Principles of Neurology 10th Edition: McGraw-Hill Education (pp 1005,1007,1016)

2. Lipka, J., Kuijpers, M., Jaworski, J. \& Hoogenraad, Casper C. (2013). Mutations in cytoplasmic dynein and its regulators cause malformations of cortical development and neurodegenerative diseases. Biochemical Society Transactions. 41(6), 1605-1612. doi: 10.1042/ bst20130188

3. Mirzaa, G.M., Conti, V., Timms, A.E., Smyser, C.D., Ahmed, S., Carter, M., Barnett, S., Hufnagel, R.B., Goldstein, A., NarumiKishimoto, Y., Olds, C., Collins, S., Johnston, K., Deleuze, J.F., Nitschke, P., Friend, K., Harris, C., Goetsch, A., Martin, B., Boyle, E.A., Parrini, E., Mei, D., Tattini, L., Slavotinek, A., Blair, E., Barnett, C., Shendure, J., Chelly, J., Dobyns, 
W.B. \& Guerrini, R. (2015). Characterisation of mutations of the phosphoinositide-3-kinase regulatory subunit, PIK3R2, in perisylvian polymicrogyria: a next-generation sequencing study. Lancet Neurol. 14(12), 1182-1195. doi: 10.1016/s1474-4422(15)00278-1.

4. Moon, H.M. \& Wynshaw-Boris, A. (2013). Cytoskeleton in action: lissencephaly, a neuronal migration disorder. Wiley Interdiscip Rev Dev Biol. 2(2), 229-245. doi: 10.1002/wdev.67.

5. Stutterd, C.A. \& Leventer, R.J. (2014). Polymicrogyria: a common and heterogeneous malformation of cortical development. Am JMed Genet C Semin Med Genet. 166C(2), 227-239. doi: 10.1002/ajmg.c.31399. 